\title{
High Current-Induced Electron Redistribution in a CVD-Grown Graphene Wide Constriction
}

\author{
Chiashain Chuang, ${ }^{1}$ Tak-Pong Woo, ${ }^{2,3}$ Fan-Hung Liu, ${ }^{4}$ Masahiro Matsunaga, \\ Yuichi Ochiai, ${ }^{1}$ Nobuyuki Aoki, ${ }^{1}$ and Chi-Te Liang ${ }^{2,4}$ \\ ${ }^{1}$ Graduate School of Advanced Integration Science, Chiba University, Chiba 263-8522, Japan \\ ${ }^{2}$ Department of Physics, National Taiwan University, Taipei 106, Taiwan \\ ${ }^{3}$ The Germination Program Office, Science \& Technology Policy Research and Information Center, NARLabs, Taipei 106, Taiwan \\ ${ }^{4}$ Graduate Institute of Applied Physics, National Taiwan University, Taipei 106, Taiwan
}

Correspondence should be addressed to Tak-Pong Woo; bonwood@gmail.com, Nobuyuki Aoki; n-aoki@faculty.chiba-u.jp, and Chi-Te Liang; ctliang@phys.ntu.edu.tw

Received 22 January 2016; Accepted 8 May 2016

Academic Editor: Yasuhiko Hayashi

Copyright (C) 2016 Chiashain Chuang et al. This is an open access article distributed under the Creative Commons Attribution License, which permits unrestricted use, distribution, and reproduction in any medium, provided the original work is properly cited.

\begin{abstract}
Investigating the charge transport behavior in one-dimensional quantum confined system such as the localized states and interference effects due to the nanoscale grain boundaries and merged domains in wide chemical vapor deposition graphene constriction is highly desirable since it would help to realize industrial graphene-based electronic device applications. Our data suggests a crossover from interference coherent transport to carriers flushing into grain boundaries and merged domains when increasing the current. Moreover, many-body fermionic carriers with disordered system in our case can be statistically described by mean-field Gross-Pitaevskii equation via a single wave function by means of the quantum hydrodynamic approximation. The novel numerical simulation method supports the experimental results and suggests that the extreme high barrier potential regions on graphene from the grain boundaries and merged domains can be strongly affected by additional hot charges. Such interesting results could pave the way for quantum transport device by supplying additional hot current to flood into the grain boundaries and merged domains in one-dimensional quantum confined CVD graphene, a great advantage for developing graphene-based coherent electronic devices.
\end{abstract}

\section{Introduction}

Graphene, a single layer of carbon atoms arranged in a hexagonal lattice, has demonstrated novel optoelectronic and electronic devices in information technology, such as photodetectors [1], solar cells [2, 3], and transistors [4]. Most importantly, the large-scale application of graphene is an urgent development for industrial developments and integrations. The chemical vapor deposition (CVD) growth of graphene has revealed great potential due to the largearea, cost-effective, and industrial-like schemes such as optical lithography to fabricate one-dimensional graphene quantum constrictions for electronic coherent devices [510]. Recent reports underscore the importance of interference effects due to the intrinsic nanoscale grain boundaries and merged domains within the CVD graphene [11, 12]. Such nanoscale grain boundaries and merged domains revealed different transport properties studied by low-temperature scanning gate microscopy (LT-SGM) for making current paths across that specific regions $[13,14]$ so as to strongly enhance the interference effect in comparison with homogenous exfoliated graphene, like backscattering suppression and Klein tunneling [15]. Such interesting CVD graphenebased electronic devices with its intrinsic grain boundaries and merged domains due to Aharonov-Bohm effect have recently revealed interesting interference effect [14] and could possibly finely control and tune the total conductance by gate voltages, magnetic fields, and source-drain currents so as to generate a high on/off ratio signal as a quantum coherent transistor for next generation information technology [16]. 


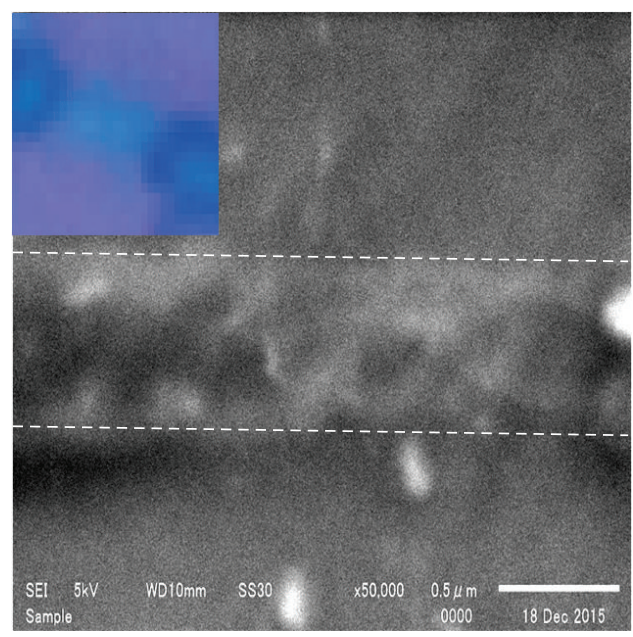

(a)

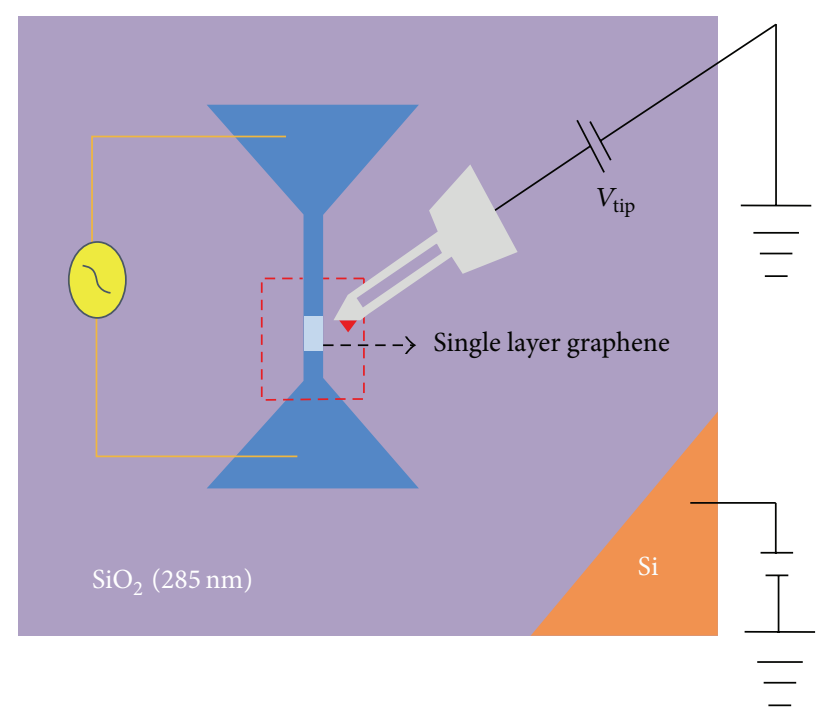

(b)

FIGURE 1: (a) Scanning electron microscopy image with $0.5 \mu \mathrm{m}$ scale bar near the single layer graphene region of the CVD graphene wide constriction. The white dash line showed the outline of single layer graphene. Inset shows the optical contrast in single layer graphene wide constriction region. (b) Electrical setup with AC source-drain current passed through the wide constriction region used to perform scanning gate microscopy with DC voltage on the tip and the height to the surface $(h \approx 200 \mathrm{~nm}$ ) for slight perturbations.

Consequently, the microscopic view of fermionic dynamic transport behaviors within wide CVD graphene constriction becomes a necessary study for realizing the applications of graphene-based electronic devices.

In this work, we used LT-SGM to study the interference behavior within a CVD graphene wide constriction region. With low current, we observed the enhanced and reduced conductance pattern outside the wide constriction region, indicative of the ring-structure current paths due to the nanoscale grain boundaries and merged domains within the wide constrictions. With additional hot current, the stimulations based on mean-field Gross-Pitaevskii equation revealed that the current charges were floored into the nanoscale grain boundaries and merged domains so as to reduce the interference effect that is consistent with our previous LT-SGM experimental results [14], which provide deep understanding of the one-dimensional CVD graphene transport and also provide a novel transport mechanism by tuning hot current into wide CVD graphene constrictions.

\section{Experimental}

High-quality and uniform commercial CVD graphene [17] on $285 \mathrm{~nm}$ thick $\mathrm{SiO}_{2} / \mathrm{Si}$ wafer was prepared, and the graphene films were known as different layer numbers and polycrystalline consisting of grains [18]. The CVD single layer graphene region, which can be confirmed by optical microscope image in green channel contract [19] as shown in Figure 1(a) inset and atomic force microscopy images as in our previous studies $[13,14]$, was particularly searched and it was etched to wide constriction with $W=800 \mathrm{~nm}$ and $L=1000 \mathrm{~nm}$ using oxygen plasma etching and deposited with two terminal Ti/Au contacts for source-drain electrodes.
Especially, the single layer region reveals nanoscale ripples due to the grain boundaries and merged domains observed by scanning electron microscopy (SEM) as shown in Figure 1(a).

Our home-built LT-SGM setup is mounted inside a $\mathrm{He}^{3}$ cryostat with a base temperature of $T=0.3 \mathrm{~K}$ and superconducting magnet of $B=9 \mathrm{~T}$. In order to sensitively perturb the carrier transport interference effect, an AC driving current from lock-in amplifiers ejects through the single layer graphene wide constriction. Furthermore, the conductance is recorded when a LT-SGM tip scans in the square region $(4.2 \mu \mathrm{m} \times 4.2 \mu \mathrm{m}$ at $1.8 \mathrm{~K})$ near the single layer graphene wide constriction with lift-mode operation at constant height $(h \approx 200 \mathrm{~nm})$ over the surface as previous studies $[13,14]$ and as shown in Figure 1(b).

\section{Results and Discussion}

As shown in Figure 2(a), negative magnetoresistance was observed at $T=1 \mathrm{~K}$ in the case that the magnetoresistance decreased with increasing $B$ near zero magnetic field, which is the evidence for the weak localization (WL) effect [2022 ]. We are able to calculate the phase coherence length by the following typical equation in disordered graphene system [23]:

$$
\begin{aligned}
& \Delta \rho(B)=-\frac{e^{2} \rho^{2}}{\pi h}\left[F\left(\frac{B}{B_{\phi}}\right)-F\left(\frac{B}{B_{\phi}+B_{i}}\right)\right. \\
& \left.-2 F\left(\frac{B}{B_{\phi}+B_{*}}\right)\right], \\
& F(z)=\ln z+\psi\left(\frac{1}{2}+\frac{1}{z}\right), \\
& B_{\phi, i, *}=\frac{\hbar c}{4 e} L_{\phi, i, *}^{-2},
\end{aligned}
$$




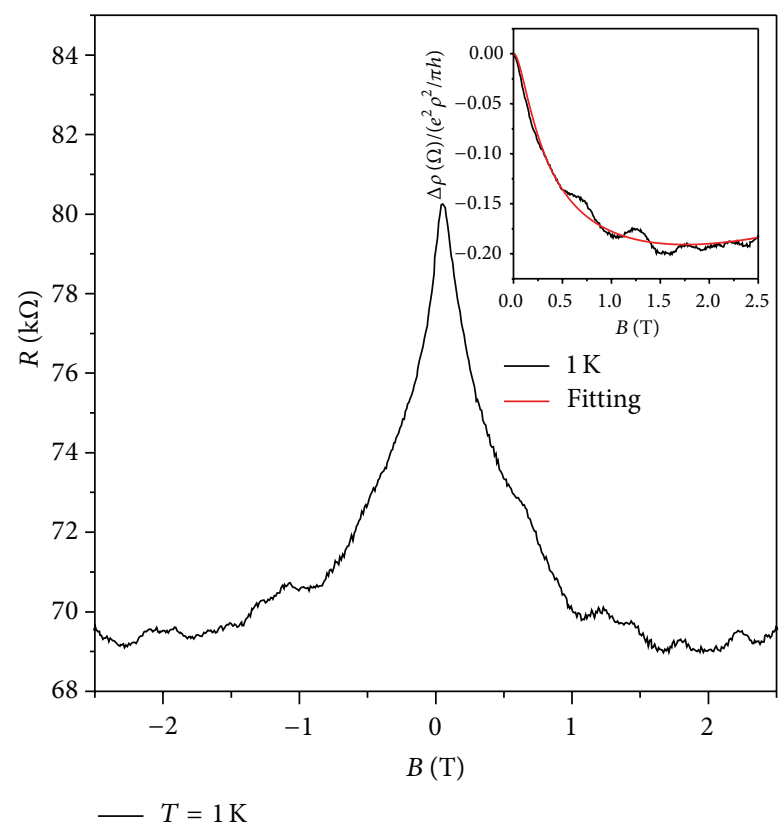

(a)

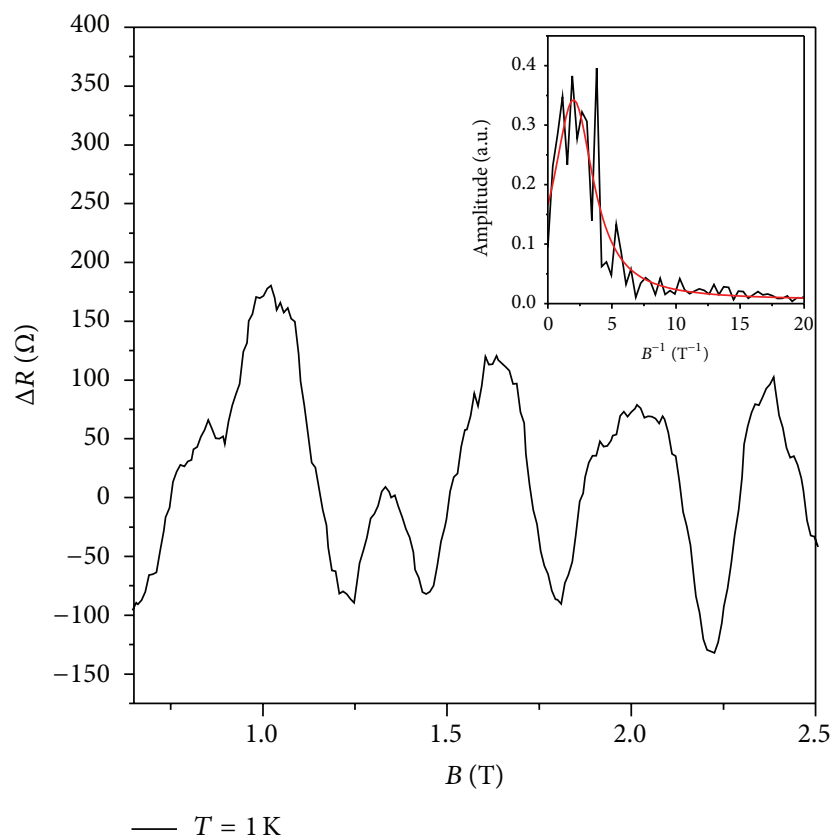

(b)

Figure 2: (a) The resistance $R(B)$ from $B=-2.5$ to $2.5 \mathrm{~T}$ through the CVD graphene wide constriction as a function of magnetic field at $T=$ $1 \mathrm{~K}$. Inset shows the WL fitting from $B=0$ to $2.5 \mathrm{~T}$ (red line) at $T=1 \mathrm{~K}$ for magnetoconductance (normalized by $e^{2} / \pi h$ ) of the wide constricted CVD graphene device. (b) The periodic resistance oscillations after eliminating the WL peak and averaging background at $T=1 \mathrm{~K}$. The inset shows the Fourier spectrum of the resistance oscillations at $T=1 \mathrm{~K}$ with the Lorentz fitting (red line) for periodic magnetic field interval $\Delta B$ $=0.49 \mathrm{~T}$.

where $\rho, \psi(z), h, e, L_{\phi}, L_{i}$, and $L_{*}$ are the magnetoresistance, digamma function, the Planck constant, electron charge, phase coherence length, intervalley scattering length, and intravalley scattering length, respectively. The inset of Figure 2(a) reveals the normalized magnetoconductance (black curve) which could be fitted well by the theoretical equation (red curve) although there are some conductance oscillations near high magnetic field regime due to the mesoscopic wide constrictions and highly disordered conditions $[13,14]$. Based on this fitting, the phase coherence length at $T=1 \mathrm{~K}$ is $L_{\phi}$ $=80 \mathrm{~nm}$, which indicated the highly disordered condition $\left(W \cong 800 \mathrm{~nm} \gg L_{\phi}\right)$ due to the atomically sharp disorder, like nanoscale grain boundaries within the CVD graphene [24].

Interestingly, we observed the periodic oscillations after eliminating from the WL peak and the average background of the resistance as shown in Figure 2(b) as previous studies [13, 14]. Consequently, the oscillation period in magnetic field is around $\Delta B=0.49 \mathrm{~T}$ for the corresponding Fourier spectrum with the peak of Lorentz fitting as shown in the inset of Figure 2(b). Due to the result of $\Delta B=0.49 \mathrm{~T}$, we are able to calculate the ring radius $r_{\mathrm{a}} \approx 52 \mathrm{~nm}(\mathrm{~A} \Delta B \sim h / e)$ based on the Aharonov-Bohm effect within our CVD graphene wide constriction region according to the grain boundaries and merged domains as previous reports $[13,14,25]$. Consistently, the $L_{\phi}=80 \mathrm{~nm}$ is at $T=1 \mathrm{~K}$ in WL effect, in good accordance with the atomically sharp disorder $\left(W \gg L_{\phi} \sim r_{\mathrm{a}}\right)$ [25].

As shown in Figure 3(a), the conductance $G\left(V_{\mathrm{bg}}\right)$ is measured by sweeping the back gate voltage from $0 \mathrm{~V}$ to $-10 \mathrm{~V}$ with $I=3 \mathrm{nA}$ and $T=1.8 \mathrm{~K}$. From the $285 \mathrm{~nm}$ thick $\mathrm{SiO}_{2}$ substrate, the field-effect mobility $\mu_{\mathrm{FE}}$ is below $100 \mathrm{~cm}^{2} / \mathrm{Vs}$ [26] and the Dirac point is far from zero voltage $(>0 \mathrm{~V})$, which is possibly due to the significant $p$-type chemical doping. Besides, there are conductance oscillations at $T=1.8 \mathrm{~K}$, which also suggests that the evidence of ring structures within wide constrictions is due to the nanoscale grain boundaries and merged domains as our previous studies $[13,14]$.

In order to further confirm the ring structures within charge transport behavior of conductance oscillations with back gate voltage, we present LT-SGM images with $V_{\mathrm{bg}}=-6 \mathrm{~V}$ at $T=1.8 \mathrm{~K}$ and zero magnetic field as shown in Figures 3(b) and 3(c). Figure 3(b) reveals electrostatic force microscopy (EFM) image for searching the graphene wide constriction region due to the charge screening effect on graphene [27]. Obviously, the graphene wide constriction region is revealed in bright color as shown in Figure 3(b). Interestingly, the enhancing and reducing conductance spots are not restricted to the wide constriction outline that was marked by white dashed line in Figure 3(c), and the hot current would suppress the conductance oscillations as previous reports $[14,25$, 28]. Such LT-SGM conductance characteristics are typical evidences for quantum ring structures due to the lateral expanse of the tip perturbation as previous reports $[13,14$, $29,30]$. From the SEM image of Figure 1(a), the single layer region was revealed to have few nanoscale ripples, which are strong evidences for the existence of nanoscale grain boundaries and merged domains so as to cause the ringstructure transport behaviors as previous studies $[13,14]$. 


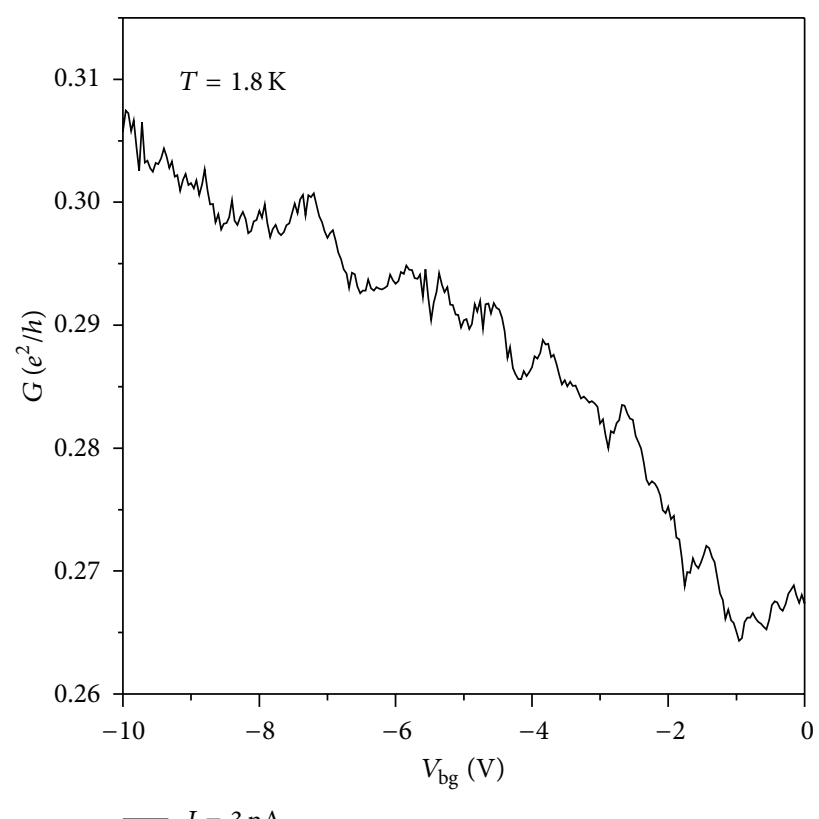

(a)

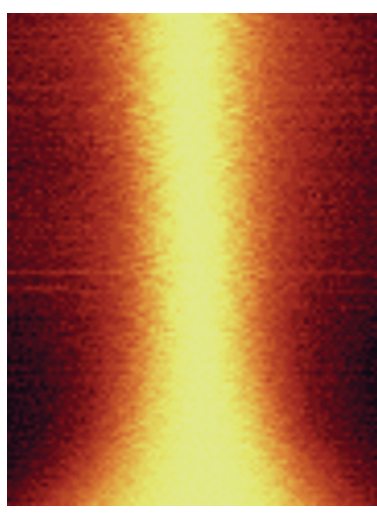

(b)

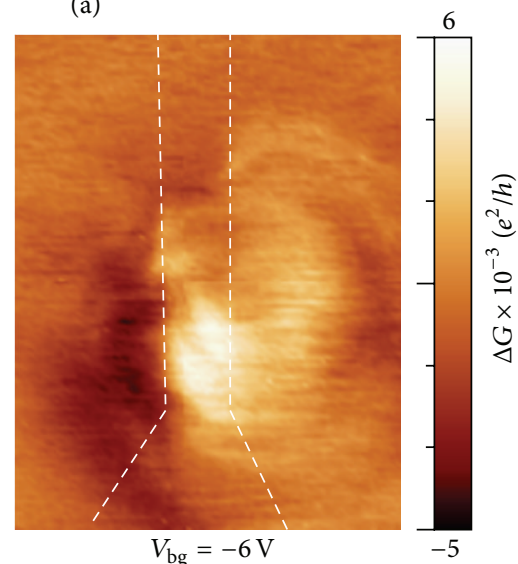

(c)

FIGURE 3: (a) The conductance in the CVD graphene wide constriction as a function of $V_{\mathrm{bg}}$ at $T=1.8 \mathrm{~K}$ from $V_{\mathrm{bg}}=0$ to $-10 \mathrm{~V}$ with source-drain current $I=3 \mathrm{nA}$. (b) Electrostatic force microscopy phase image of the graphene device, with $V_{\text {tip }}=2 \mathrm{~V}$ and at $T=1.8 \mathrm{~K}$, which obviously shows the single layer graphene wide constriction region. (c) Lift-mode low-temperature scanning gate images with back gate voltage $V_{\mathrm{bg}}=$ $-6 \mathrm{~V}$ and $T=1.8 \mathrm{~K}$. The white dash line shows the outline of single layer graphene wide constriction region.

Most importantly, the charge quantum transport dynamic evolution with increase of the current so as to suppress the interference effect within the CVD graphene wide constriction has never been studied to date although some experimental results have observed the suppression of interference effect in ring structures devices [14, 25, 31]. We are interested in whether the additional carriers flushing would not follow the nanoscale ring-structure paths. Therefore, we performed numerical simulations based on the mean-field Gross-Pitaevskii equation [32-35] (see Supporting Information in Supplementary Material available online at http://dx.doi.org/10.1155/2016/1806871 for detail). The unit one normalized charge density $\left(n / n_{0}\right)$ is defined by ideal charge distribution without any disordered potential fluctuations. Considering the disordered potential fluctuation that comes from the nanoscale grain boundaries and merged domains, we used autocorrelation function to set up the initial condition of the original disordered potential distribution in $800 \mathrm{~nm}$ (width) $\times 1000 \mathrm{~nm}$ (length) region from the variation of conductance fluctuation distribution as shown in Figure 3(c) so as to simulate the normalized charge density distribution in a tiny steady unit AC current $I_{0}$ without affecting the disordered condition that is intrinsic barrier potentials from nanoscale ring structures as shown in Figure 4(a) [29]. By increasing the current to $300 I_{0}, 500 I_{0}$, $700 I_{0}$, and $900 I_{0}$ as shown in Figures 4(b)-4(e), the normalized charge density fluctuation distribution in this region gradually becomes small and the normalized charge density 


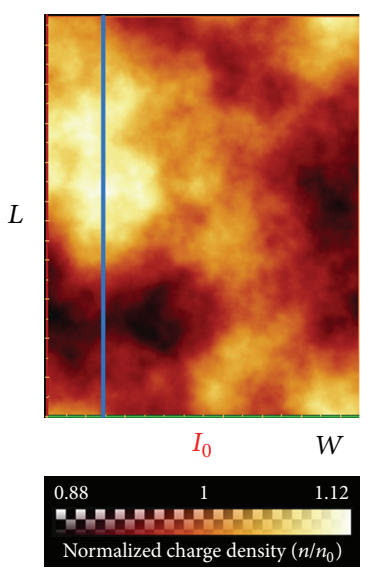

(a)

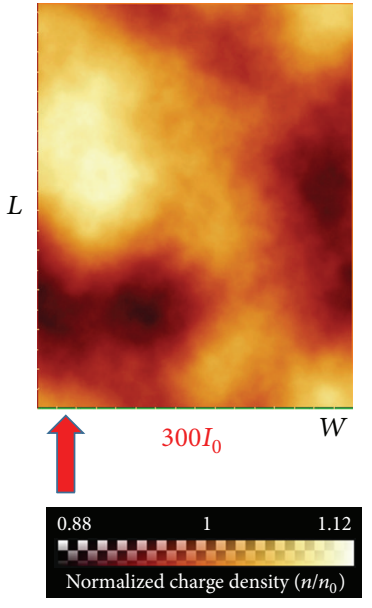

(b)

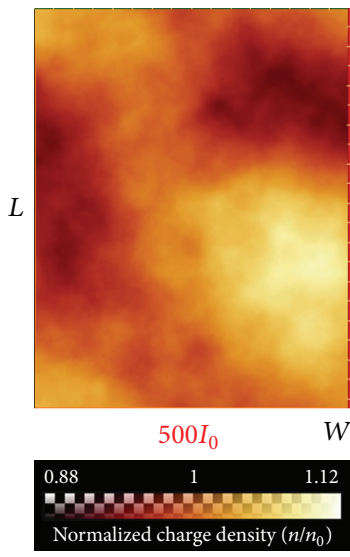

(c)

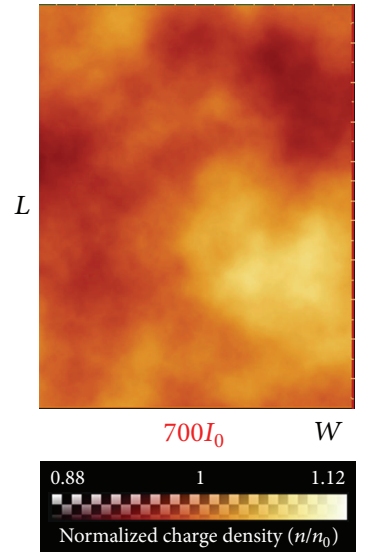

(d)
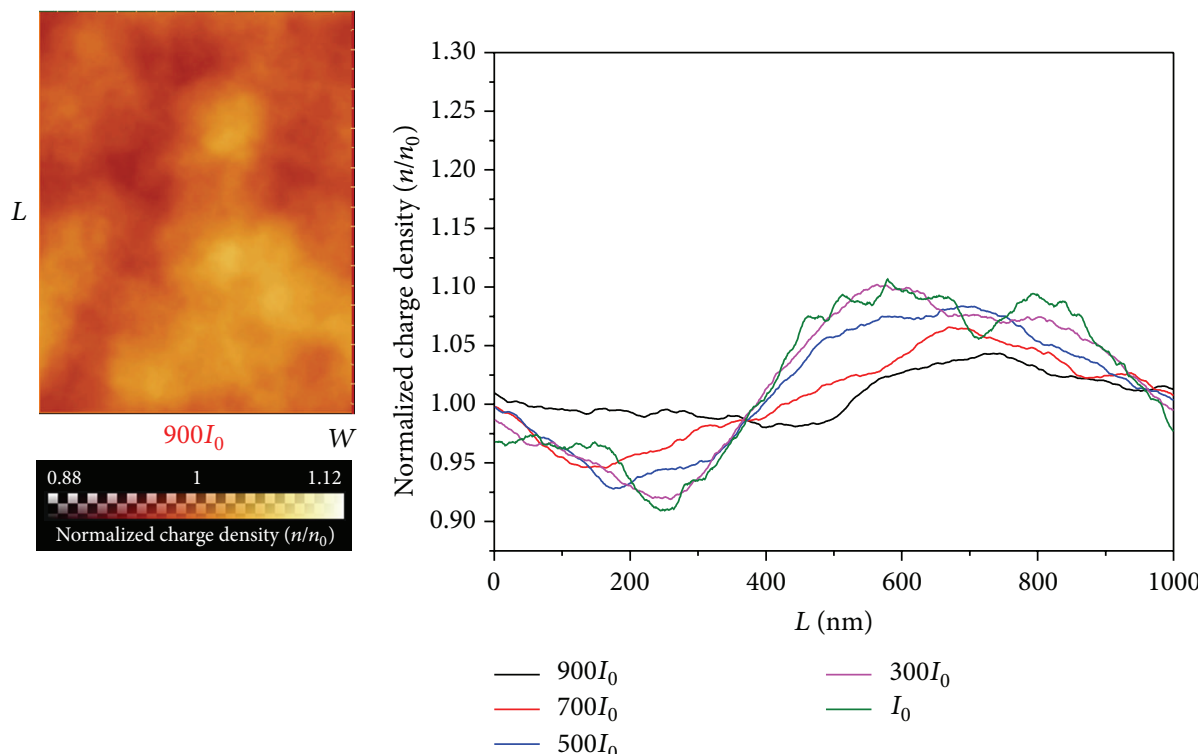

(e)

(f)

FIGURE 4: The simulated images of the change in normalized charge density in the area $(L=1000 \mathrm{~nm} \times W=800 \mathrm{~nm})$ obtained at the currents (a) $I=I_{0}$, (b) $I=300 I_{0}$, (c) $I=500 I_{0}$, (d) $I=700 I_{0}$, and (e) $I=900 I_{0}$. The red arrow in (b) reveals the current ejected direction that is parallel to the wide constriction length $L$. (f) The line profile (blue line marked in (a)) of the normalized charge density and the length $L$ with different ejected currents $I=I_{0}, 300 I_{0}, 500 I_{0}$, and $700 I_{0}$.

gradually becomes unit one supported by the line profile (blue line shown in Figure 4(a)) as shown in Figure 4(f), which indicates that the increasing hot current would start to flush into the barrier potentials so as to suppress the interference effect, an excellent correspondence to previous experimental results $[14,25,31]$. From this point of view, the hot charges with spreading kinetic energy would flush into the barrier potentials that are equivalent to nanoscale ring structures within the wide constriction under supply of the additional hot charge, which interestingly implies that hot fermions in single layer graphene could flush into the barrier potentials formed from CVD graphene grain boundaries and merged domains that is a novel transport property in comparison with the serious scattering fermions in low current regime by grain boundaries and merged domains (see Electronic Supplementary Material for a movie of the evolution [36]). Our results obtained on CVD graphene in wide constriction show that the interference effect is sensitive in the low current regime due to the intrinsic nanoscale ring structures, and the interference effect is suppressed in high current regime. The LT-SGM experiments and simulation results reveal that the hot charges start to flush into the nanoscale grain boundaries and merged domains by supplying additional hot charges into the wide constriction region, which is a novel 
transport mechanism in comparison with conventional twodimensional electron gas systems [37-41].

\section{Conclusions}

In conclusion, we performed LT-SGM experiments on CVD-grown graphene wide constriction, which was further supported by numerical simulations. The LT-SGM images revealed the nanoscale ring structures existence so as to cause interference effect. Meanwhile, the numerical simulations suggested that the hot charges could flush into the grain boundaries and merged domains from the dynamical point of view. This work provides a subtle unified scope of current dependence charge transport behavior and pictures on CVD graphene in wide constriction, a great advance for realizing industrial graphene-based electronic device.

\section{Competing Interests}

The authors declare that they have no competing interests.

\section{Acknowledgments}

As an International Research Fellow of the Japan Society for the Promotion of Science (JSPS), Chiashain Chuang acknowledges the JSPS Postdoctoral Fellowship.

\section{References}

[1] S. Lara-Avila, K. Moth-Poulsen, R. Yakimova et al., "Nonvolatile photochemical gating of an epitaxial graphene/polymer heterostructure," Advanced Materials, vol. 23, no. 7, pp. 878-882, 2011.

[2] X. Wang, L. Zhi, and K. Müllen, "Transparent, conductive graphene electrodes for dye-sensitized solar cells," Nano Letters, vol. 8, no. 1, pp. 323-327, 2008.

[3] X. Li, H. Zhu, K. Wang et al., "Graphene-on-silicon schottky junction solar cells," Advanced Materials, vol. 22, no. 25, pp. 2743-2748, 2010.

[4] F. Xia, T. Mueller, R. Golizadeh-Mojarad et al., "Photocurrent imaging and efficient photon detection in a graphene transistor," Nano Letters, vol. 9, no. 3, pp. 1039-1044, 2009.

[5] A. Reina, X. Jia, J. Ho et al., "Large area, few-layer graphene films on arbitrary substrates by chemical vapor deposition," Nano Letters, vol. 9, no. 1, pp. 30-35, 2009.

[6] V. Dhand, K. Y. Rhee, H. J. Kim, and D. H. Jung, "A comprehensive review of graphene nanocomposites: research status and trends," Journal of Nanomaterials, vol. 2013, Article ID 763953, 14 pages, 2013.

[7] S. Bae, H. Kim, Y. Lee et al., "Roll-to-roll production of 30-inch graphene films for transparent electrodes," Nature Nanotechnology, vol. 5, no. 8, pp. 574-578, 2010.

[8] Y.-P. Hsieh, Y.-W. Wang, C.-C. Ting, H.-C. Wang, K.-Y. Chen, and C.-C. Yang, "Effect of catalyst morphology on the quality of CVD grown graphene," Journal of Nanomaterials, vol. 2013, Article ID 393724, 6 pages, 2013.

[9] C. Chuang, L.-H. Lin, N. Aoki et al., "Experimental evidence for direct insulator-quantum Hall transition in multi-layer grapheme," Nanoscale Research Letters, vol. 8, article 214, pp. 15, 2013.
[10] C.-T. Liang, M. Y. Simmons, C. G. Smith, G. H. Kim, D. A. Ritchie, and M. Pepper, "Spin-dependent transport in a clean one-dimensional channel," Physical Review B, vol. 60, no. 15, pp. 10687-10690, 1999.

[11] J. C. Koepke, J. D. Wood, D. Estrada et al., "Atomic-scale evidence for potential barriers and strong carrier scattering at graphene grain boundaries: a scanning tunneling microscopy study," ACS Nano, vol. 7, no. 1, pp. 75-86, 2013.

[12] Y. Ogawa, K. Komatsu, K. Kawahara, M. Tsuji, K. Tsukagoshi, and H. Ago, "Structure and transport properties of the interface between CVD-grown graphene domains," Nanoscale, vol. 6, no. 13, pp. 7288-7294, 2014.

[13] C. Chuang, M. Matsunaga, F.-H. Liu et al., "Probing weak localization in chemical vapor deposition graphene wide constriction using scanning gate microscopy," Nanotechnology, vol. 27, no. 7, Article ID 075601, 2016.

[14] C. Chuang, M. Matsunaga, F.-H. Liu et al., "Imaging coherent transport in chemical vapor deposition graphene wide constriction using scanning gate micrscopy," Applied Physics Letters, vol. 108, no. 12, Article ID 123105, 2016.

[15] N. Stander, B. Huard, and D. Goldhaber-Gordon, "Evidence for Klein tunneling in graphene p-n junctions," Physical Review Letters, vol. 102, no. 2, Article ID 026807, 2009.

[16] J. Munárriz, F. Domínguez-Adame, and A. V. Malyshev, "Toward graphene-based quantum interference devices," Nanotechnology, vol. 22, no. 36, Article ID 365201, 2011.

[17] X. Li, W. Cai, J. An et al., "Large-area synthesis of high-quality and uniform graphene films on copper foils," Science, vol. 324, no. 5932, pp. 1312-1314, 2009.

[18] https://graphene-supermarket.com/Multilayer-Graphene-on285-nm-Silicon-Dioxide-Wafer-10-pack.html.

[19] C. Casiraghi, A. Hartschuh, E. Lidorikis et al., "Rayleigh imaging of graphene and graphene layers," Nano Letters, vol. 7, no. 9, pp. 2711-2717, 2007.

[20] S. V. Morozov, K. S. Novoselov, M. I. Katsnelson et al., "Strong suppression of weak localization in graphene," Physical Review Letters, vol. 97, no. 1, Article ID 016801, 2006.

[21] F.-H. Liu, C.-S. Hsu, C. Chuang et al., "Dirac fermion heating, current scaling, and direct insulator-quantum hall transition in multilayer epitaxial graphene," Nanoscale Research Letters, vol. 8, no. 1, pp. 1-6, 2013.

[22] C. Chuang, T.-P. Woo, A. M. Mahjoub et al., "Weak localization and universal conductance fluctuations in multi-layer graphene," Current Applied Physics, vol. 14, no. 1, pp. 108-111, 2014.

[23] E. McCann, K. Kechedzhi, V. I. Fal'ko, H. Suzuura, T. Ando, and B. L. Altshuler, "Weak-localization magnetoresistance and valley symmetry in graphene," Physical Review Letters, vol. 97, no. 14, Article ID 146805, 2006.

[24] H. Cao, Q. Yu, L. A. Jauregui et al., "Electronic transport in chemical vapor deposited graphene synthesized on $\mathrm{Cu}$ : quantum Hall effect and weak localization," Applied Physics Letters, vol. 96, no. 12, Article ID 122106, 2010.

[25] S. Russo, J. B. Oostinga, D. Wehenkel et al., "Observation of Aharonov-Bohm conductance oscillations in a graphene ring," Physical Review B, vol. 77, no. 8, Article ID 085413, 2008.

[26] K. S. Novoselov, A. K. Geim, S. V. Morozov et al., "Electric field effect in atomically thin carbon films," Science, vol. 306, no. 5696, pp. 666-669, 2004.

[27] S. S. Datta, D. R. Strachan, E. J. Mele, and A. T. C. Johnson, "Surface Potentials and layer charge distributions in few-layer graphene films," Nano Letters, vol. 9, no. 1, pp. 7-11, 2009. 
[28] C. Chuang, L.-H. Lin, N. Aoki et al., "Mesoscopic conductance fluctuations in multi-layer graphene," Applied Physics Letters, vol. 103, no. 4, Article ID 043117, 2013.

[29] D. Cabosart, S. Faniel, F. Martins et al., "Imaging coherent transport in a mesoscopic graphene ring," Physical Review B: Condensed Matter and Materials Physics, vol. 90, Article ID 205433, 2014.

[30] B. Hackens, F. Martins, T. Ouisse et al., "Imaging and controlling electron transport inside a quantum ring," Nature Physics, vol. 2, no. 12, pp. 826-830, 2006.

[31] S. Washburn and R. A. Webb, "Aharonov-Bohm effect in normal metal quantum coherence and transport," Advances in Physics, vol. 35, no. 4, pp. 375-422, 1986.

[32] G. Manfredi and F. Haas, "Self-consistent fluid model for a quantum electron gas," Physical Review B, vol. 64, no. 7, Article ID 075316, 2001.

[33] P.-H. Chavanis, "Mass-radius relation of Newtonian selfgravitating Bose-Einstein condensates with short-range interactions. I. Analytical results," Physical Review D, vol. 84, no. 4, Article ID 043531, 2011.

[34] T.-P. Woo and T. Chiueh, "High-resolution simulation on structure formation with extremely light bosonic dark matter," The Astrophysical Journal, vol. 697, no. 1, pp. 850-861, 2009.

[35] G. Manfredi and F. Haas, "Self-consistent fluid model for a quantum electron gas," Physical Review B: Condensed Matter and Materials Physics, vol. 64, Article ID 075316, 2001.

[36] https://www.youtube.com/watch?v=JW7-oEGsQJQ.

[37] J.-H. Chen, J.-Y. Lin, J.-K. Tsai et al., "Experimental evidence for Drude-Boltzmann-like transport in a two-dimensional electron gas in an $\mathrm{AlGaN} / \mathrm{GaN}$ heterostructure," Journal of the Korean Physical Society, vol. 48, no. 6, pp. 1539-1543, 2006.

[38] J. R. Juang, T.-Y. Huang, T.-M. Chen et al., "Transport in a gated $\mathrm{Al}_{0.18} \mathrm{Ga}_{0.82} \mathrm{~N} / \mathrm{GaN}$ electron system," Journal of Applied Physics, vol. 94, no. 5, pp. 3181-3184, 2003.

[39] K. S. Cho, T.-Y. Huang, C.-P. Huang et al., "Exchange-enhanced $g$-factors in an $\mathrm{Al}_{0.25} \mathrm{Ga}_{0.75} \mathrm{~N} / \mathrm{GaN}$ two-dimensional electron system," Journal of Applied Physics, vol. 96, no. 12, pp. 7370-7373, 2004.

[40] D. R. Hang, C.-T. Liang, J.-R. Juang et al., "Electrically detected and microwave-modulated Shubnikov-de Haas oscillations in an $\mathrm{Al}_{0.4} \mathrm{Ga}_{0.6} \mathrm{~N} / \mathrm{GaN}$ heterostructure," Journal of Applied Physics, vol. 93, no. 4, pp. 2055-2058, 2003.

[41] D. R. Hang, C.-T. Liang, C. F. Huang et al., "Effective mass of two-dimensional electron gas in an $\mathrm{Al}_{0.2} \mathrm{Ga}_{0.8} \mathrm{~N} / \mathrm{GaN}$ heterojunction," Applied Physics Letters, vol. 79, no. 1, pp. 66-68, 2001. 

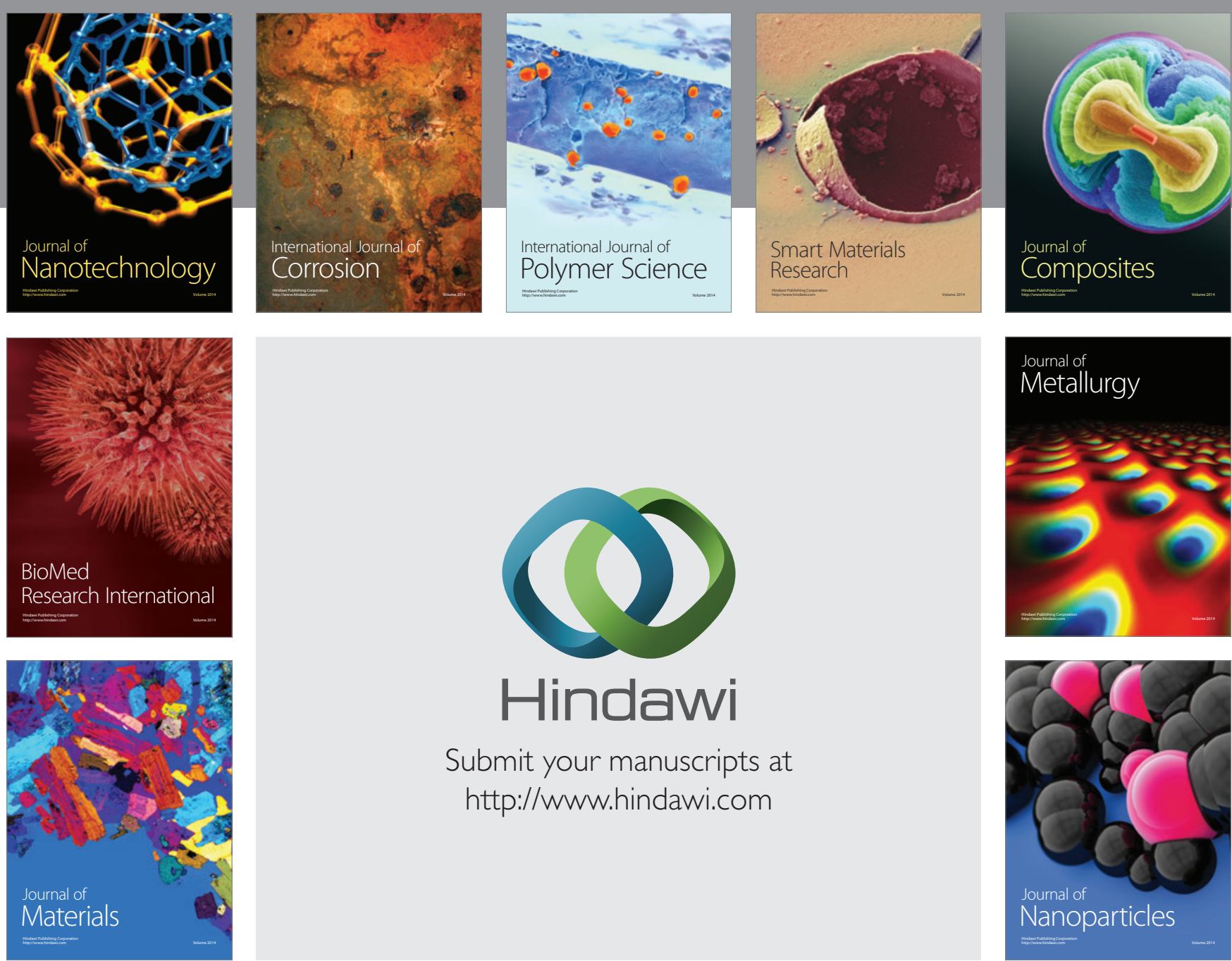

\section{Hindawi}

Submit your manuscripts at

http://www.hindawi.com

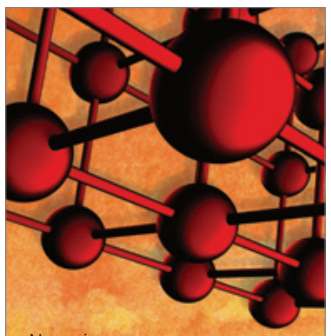

Materials Science and Engineering
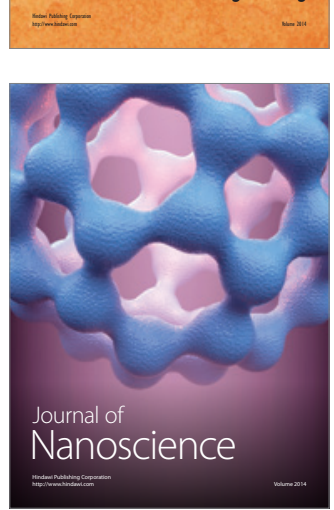
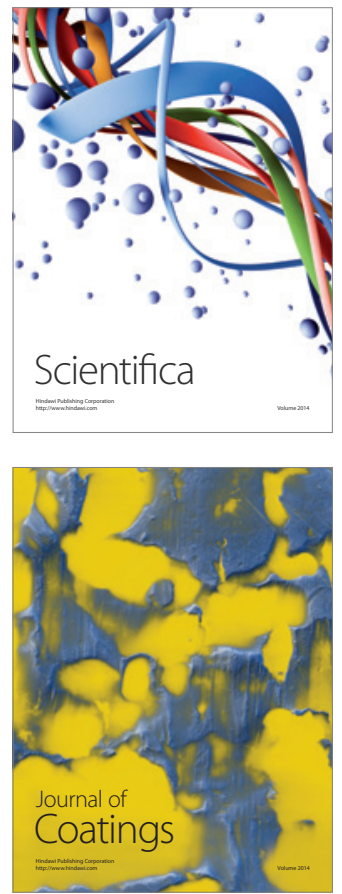
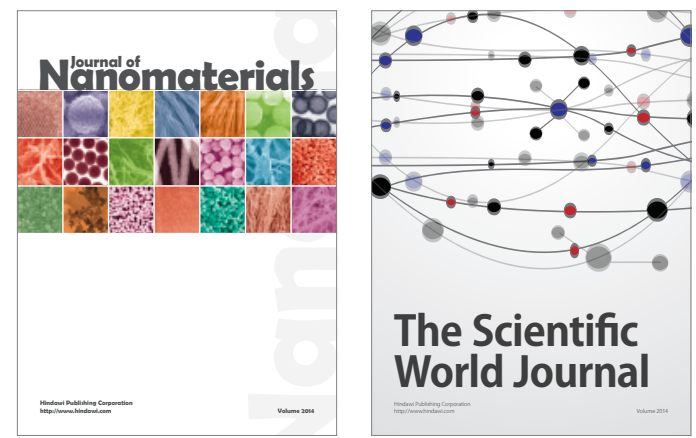

The Scientific World Journal
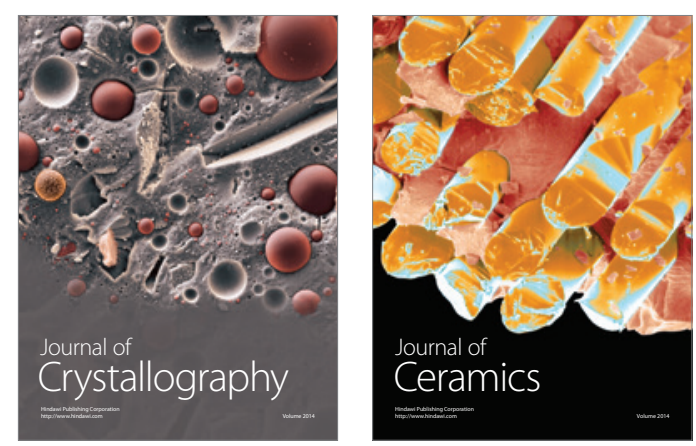
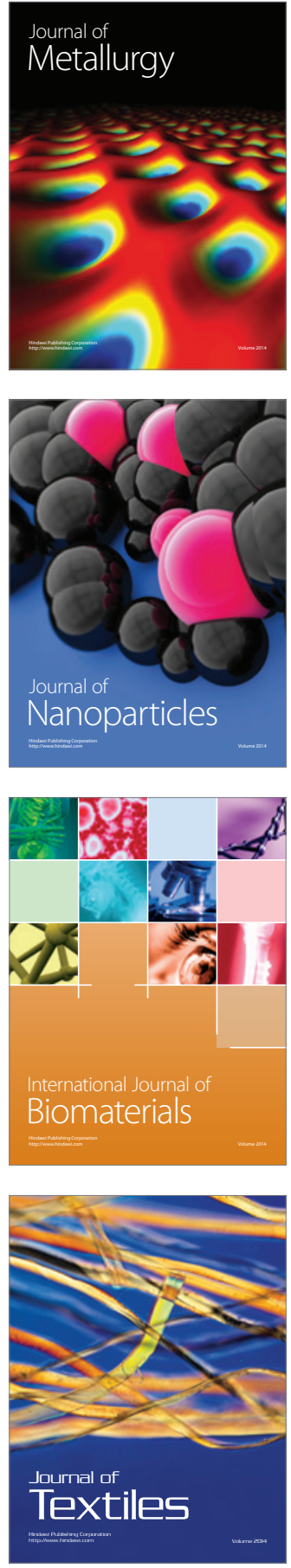